\title{
Disentangling hadronic from leptonic emission in the composite SNR G326.3-1.8
}

\author{
Justine Devin ${ }^{1}$, Fabio Acero ${ }^{2}$, Jean Ballet ${ }^{2}$ \\ on behalf of the Fermi-LAT collaboration \\ ${ }^{1}$ Laboratoire Univers et Particules de Montpellier, CNRS/IN2P3, France \\ email: justine.devin@etu.umontpellier.fr \\ ${ }^{2}$ AIM, CEA/CNRS/Paris 7, DRF/IRFU/DAp, CEA Saclay, France \\ email: fabio.acero@cea.fr or jean.ballet@cea.fr
}

\begin{abstract}
G326.3-1.8 (also known as MSH 15-56) has been detected in radio as a middle-aged composite supernova remnant (SNR) consisting of a SNR shell and a pulsar wind nebula (PWN) which has been crushed by the reverse shock. With the recent Fermi-LAT data release Pass 8 providing increased acceptance and angular resolution, we investigate the morphology of this SNR to disentangle the PWN from the SNR contributions and understand the nature of the $\gamma$-ray emission. We thus perform a morphological and spectral analysis from $300 \mathrm{MeV}$ to 300 $\mathrm{GeV}$ which highlights the contributions from these two components. The simplest interpretation is hadronic emission from the SNR and harder leptonic emission from the PWN.
\end{abstract}

Keywords. supernova remnants, gamma-rays: observations, acceleration of particles, cosmic rays

\section{Introduction}

Supernova remnants (SNRs) and pulsar wind nebulae (PWNe) have long been considered potential sources of Galactic cosmic rays and have therefore been investigated over a wide range of energies. With a SNR shell and a PWN seen at radio wavelengths, the Galactic SNR G326.3-1.8 is a prototype of the so-called composite SNRs (Mills et al. 1961). Its distance is estimated between $3.1 \mathrm{kpc}$ (Goss et al. 1972) and $4.1 \mathrm{kpc}$ (Rosado et al. 1996) as established by the $\mathrm{HI}$ absorption profile and $\mathrm{H} \alpha$ velocity measurements respectively. Temim et al. (2013) estimated the SNR to be 16,500 years old with a shock velocity of $500 \mathrm{~km} \mathrm{~s}^{-1}$. Figure 1, obtained from radio observations (Whiteoak \& Green 1996), shows a symmetric SNR shell with a $0.3^{\circ}$ radius and a PWN which has been displaced from the geometric center of the SNR and likely crushed by the reverse shock. The associated pulsar has not been detected but Chandra maps have revealed a point source embedded in the X-ray PWN located in the South West of the radio nebula (Temim et al. 2013). At higher energies, the origin of the $\gamma$-ray emission remains uncertain (Temim et al. 2013) and SNR G326.3-1.8 has recently been found to be extended with the Fermi Large Area Telescope (LAT) data (Acero et al. 2016). In this proceeding, we revisit the morphology of this SNR, with the latest data release Pass 8 of the FermiLAT (Atwood et al. 2013), and we also report a spectral analysis of our best model using two spatial components for the $\gamma$-ray emission.

\section{Data analysis}

The LAT on board the Fermi satellite is a pair conversion instrument sensitive to $\gamma$ rays in the energy range from $30 \mathrm{MeV}$ to more than $300 \mathrm{GeV}$. The new data release Pass 


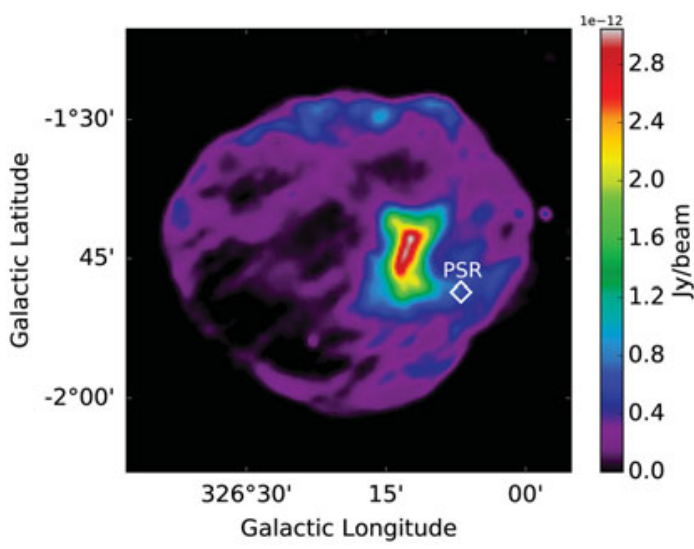

Figure 1. $843 \mathrm{MHz}$ MOST radio image of SNR G326.3-1.8 (Whiteoak \& Green 1996). The position of the pulsar candidate is represented by a white diamond.

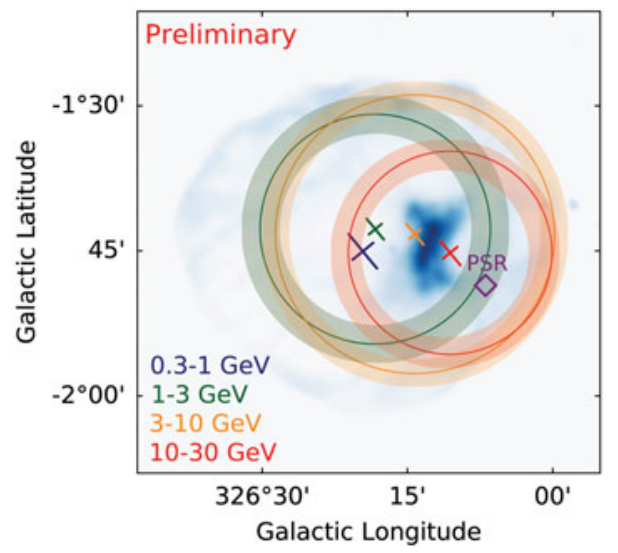

Figure 2. Best-fit Gaussians in 4 energy bands. Crosses: position uncertainties, circles: $r_{68}$ of the Gaussian (if the source is extended), shaded areas: errors on size.

8 allows for event selection based on the quality of the reconstruction of their direction, resulting in four different Point Spread Function (PSF) classes. To avoid as much as possible the contamination between the PWN and the SNR, we keep the data having the best angular resolution $\left(0.4^{\circ}\right.$ at $1 \mathrm{GeV}$ compared to $0.8^{\circ}$ with no selection) at the cost of a factor four reduction of the effective area. We perform a binned analysis from $300 \mathrm{MeV}$ to $300 \mathrm{GeV}$ using 6.5 years of data collected from August 4, 2008 to January 31, 2015, within a $10^{\circ} \times 10^{\circ}$ region around the position of SNR G326.3-1.8. We use a Test Statistic (TS) which is defined to test the likelihood of one hypothesis $\mathcal{L}_{1}$ (including a source) against the null hypothesis $\mathcal{L}_{0}$ (absence of source), such as: TS $=2 \times\left(\log \mathcal{L}_{1}-\log \mathcal{L}_{0}\right)$. This can be directly interpreted in terms of significance of hypothesis 1 with respect to the null hypothesis 0 since the TS follows a $\chi^{2}$-law for $n$ additional parameters. When adding a second component, the model with one component becomes the null hypothesis to test the significance of the second component. We first model the region starting with the 3FGL sources catalog (Acero et al. 2015) based on the previous Pass 7 data, and we add eleven sources at the positions where the significance exceeds $5 \sigma$.

\subsection{Morphological analysis}

To investigate the source morphology, we divide the data into four logarithmically spaced energy bins that we subsequently fit individually using a 2D-symmetric Gaussian. Since the PSF changes over half a decade, we need to specify the source spectrum. We parametrize it with a power law with free spectral index. The normalizations of the source, the Galactic and isotropic diffuse emissions are let free while the other sources are fixed to their best-fit values. Figure 2 depicts the results of the fitting procedure in the four energy bands. At low energies $(300 \mathrm{MeV}-1 \mathrm{GeV})$, the PSF $\left(0.4^{\circ}\right.$ at $\left.1 \mathrm{GeV}\right)$ is larger than the SNR radius and we cannot measure the extension. The centroid of the Gaussian lies outside the nebula. Between 1 and $3 \mathrm{GeV}$, the significance of the extension is more than $5 \sigma$ and the position of the Gaussian appears to be fairly consistent with the center of the radio SNR. At higher energies (from 3 to $30 \mathrm{GeV}$ ), the $\gamma$-ray morphology is still significantly extended (more than $5 \sigma$ ) and shows a clear trend toward the position of the radio PWN.

This energy-dependent source morphology clearly requires a more detailed investigation, beyond a one-component modeling. Since the PSF below $1 \mathrm{GeV}$ is not good enough 


\begin{tabular}{l|ccc}
\hline \hline Spatial models & $\mathrm{TS}$ & $\mathrm{N}_{\text {dof }}$ & $\mathrm{TS}_{P W N}$ \\
\hline radio PWN & 593.38 & 2 & - \\
\hline disk & 681.78 & 5 & - \\
disk + radio PWN & 694.81 & 7 & 13.03 \\
\hline radio SNR & 667.32 & 2 & - \\
radio SNR + radio PWN & 682.97 & 4 & 15.65 \\
\hline SNR mask & 670.32 & 2 & - \\
SNR mask + radio PWN & 696.36 & 4 & 26.04 \\
\hline
\end{tabular}

Table 1. TS values for different spatial models fitted from $1 \mathrm{GeV}$ to $300 \mathrm{GeV}$. The corresponding number of degrees of freedom $\mathrm{N}_{d o f}$ is also given (spatial and/or spectral). $\operatorname{TS}_{P W N}$ quantifies the improvement of the fit when adding the PWN component to each of the one-component models.

to resolve the SNR, the following morphological analysis uses data between 1 and 300 $\mathrm{GeV}$ and we describe the spectral models associated to each component by a power law. We first model the $\gamma$-ray emission assuming it comes only from the PWN, with its radio template (see Figure 1) and we perform a likelihood fit where the spectral parameters of the PWN as well as those of the Galactic and isotropic emissions are free during the fit. Table 1 reports the corresponding TS value. To model the contribution of an additional component which seems to be responsible for the low energy part (as depicted in Figure 2), we test several templates using first a simple disk component and then physically motivated templates (derived from the radio map of the SNR). We thus use the radio map replacing the contribution of the nebula by the average value of the radio emission around it (labeled "radio SNR" in Table 1). From that, we also create another template, following the radio shock and filled homogeneously (called "SNR mask" in Table 1). The results from our maximum likelihood fit are given in Table 1 . The TS values obtained using the one-component models (disk, radio SNR and SNR mask) alone are clearly higher than the one obtained using only the radio PWN, indicating that the fit prefers a model more extended than the radio nebula. In Table 1, we compare the TS values obtained with each of the one-component models to the two-component models testing the significance of adding the radio template of the nebula $\left(\mathrm{TS}_{P W N}\right.$ value for two degrees of freedom). For all our models, the significance of adding the PWN is between 3 and $4 \sigma$ and the maximum TS value is obtained for the model including the radio PWN with either the disk or the SNR mask. In terms of significance, our best model involves the SNR mask and the radio PWN since it requires fewer free parameters during the fit than the disk whose spatial components have been optimized.

\subsection{Spectral analysis}

To understand the underlying emission processes, we perform a spectral analysis from 300 $\mathrm{MeV}$ to $300 \mathrm{GeV}$ using our best two-component model: the SNR mask with the radio PWN. Modeling both by a power law, the fit leads to a spectral separation between the two components: a softer spectrum for the remnant $(\Gamma=2.17 \pm 0.06$ being the photon index) and a harder spectrum for the nebula $(\Gamma=1.79 \pm 0.12)$. The associated Spectral Energy Distributions (SEDs), shown in Figure 3, clearly emphasize that two distinct morphologies give rise to two distinct spectral signatures. The TS values of the components in each energy bin highlight their different contributions: at low energy $(E<10 \mathrm{GeV})$, the emission is dominated by the SNR while the contribution of the PWN protrudes above $10 \mathrm{GeV}$. If both the SNR and the PWN contribute to the $\gamma$-ray 


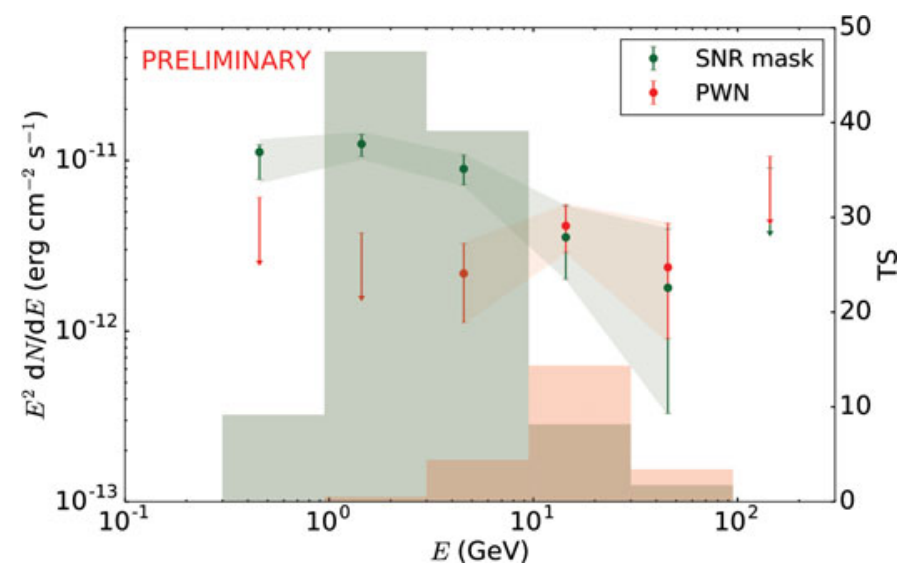

Figure 3. SED (data points) and TS values (histograms) in individual energy bands obtained with the model including the SNR mask and the radio PWN. The shaded areas represent the quadratic sum of statistical and systematic errors. The systematic errors are calculated using 8 alternative Galactic diffuse emissions as explained in the first Fermi-LAT supernova remnant catalog (Acero et al. 2016).

emission, their spectral shapes are consistent with two different underlying processes, namely hadronic and leptonic emission, respectively. Even if no molecular cloud has been reported close to this SNR, the detection of $\mathrm{H} \alpha$ emission in several regions (Van den Bergh 1979) is an evidence of interaction of the shock with dense material, therefore providing a large density of targets to produce a significant amount of hadronic emission.

\section{Conclusions}

We perform an analysis from $300 \mathrm{MeV}$ to $300 \mathrm{GeV}$ of the composite SNR G326.3-1.8 with the Fermi-LAT Pass 8 data. We find that the $\gamma$-ray morphology evolves with energy and shrinks to the radio nebula at high energies $(\mathrm{E}>10 \mathrm{GeV})$. We thus investigate a more detailed morphology using the radio map of the PWN and other models for an additional contribution such as an uniform disk, the radio map of the remnant and its radio template filled homogeneously, called here the SNR mask. Using the maximum likelihood fitting procedure, our best model involves the SNR mask and the radio PWN for which we obtain two different spectral signatures: a softer spectrum for the remnant $(\Gamma=2.17 \pm 0.06)$ and a harder spectrum for the nebula $(\Gamma=1.79 \pm 0.12)$. Their spectral shapes are consistent with hadronic and leptonic origins for the SNR and the PWN respectively and we are currently investigating the physical properties of this source.

\section{Acknowledgements}

The Fermi-LAT Collaboration acknowledges support for LAT development, operation and data analysis from NASA and DOE (United States), CEA/Irfu and IN2P3/CNRS (France), ASI and INFN (Italy), MEXT, KEK, and JAXA (Japan), and the K.A. Wallenberg Foundation, the Swedish Research Council and the National Space Board (Sweden). Science analysis support in the operations phase from INAF (Italy) and CNES (France) is also gratefully acknowledged. This work performed in part under DOE Contract DEAC02-76SF00515.

Many thanks to the organizing committees and for the financial support obtained through an IAU grant. 


\section{References}

Acero, F., Ackermann, M., Ajello, M., et al. 2015, ApJS, 218, 23

Acero, F., Ackermann, M., Ajello, M., et al. 2016, ApJS, 224, 8

Atwood, W., Albert, A., Baldini, L., et al. 2013, ArXiv e-prints

Goss, W. M., Radhakrishnan, V., Brooks, J. W., \& Murray, J. D. 1972, ApJS, 24, 123

Mills, B. Y., Slee, O. B., \& Hill, E. R. 1961, Australian Journal of Physics, 14, 497

Rosado, M., Ambrocio-Cruz, P., Le Coarer, E., \& Marcelin, M. 1996, A \& A, 315, 243

Temim, T., Slane, P., Castro, D., et al. 2013, ApJ, 768, 61

Van den Bergh, S. 1979, ApJ, 227, 497

Whiteoak, J. B. Z. \& Green, A. J. 1996, A\&AS, 118, 329 